hep-ph/0104058

\title{
On predictions of the first results from RHIC
}

\author{
Kari J. Eskola ${ }^{a}$
}

${ }^{a}$ Department of Physics, University of Jyväskylä, P.O. Box 35, FIN-40351 Jyväskylä, Finland

In this talk, I will discuss the predictions of the first results from RHIC: the charged particle multiplicity $d N_{\mathrm{ch}} / d \eta$, its centrality dependence and the elliptic flow $v_{2}$.

\section{Charged particle multiplicity in central collisions}

\subsection{Predictions}

To predict particle multiplicities in $p p$ and $A A$ collisions from first principles has so far been impossible. Consequently, various models have been introduced. In Fig. 1 I have collected predictions for the charged particle multiplicity $d N_{\mathrm{ch}} / d y$ at $y=0$ for $\mathrm{Au}-\mathrm{Au}$ collisions at $\sqrt{s}=200 \mathrm{AGeV}$, presented at Quark Matter '99 [1] and after, until July 2000 , i.e. prior to the release of the very first data from RHIC. From the top, the figure shows predictions from event generator models Heavy Ion Jet InteractioN event Generator [2], HIJING+Zhang's Parton Cascade+A Relativistic Transport model [3], Relativistic Quantum Molecular Dynamics [4], Ultrarelativistic Quantum Molecular Dynamics [5], VNI+UrQMD [6], Hadron String Dynamics and VNI+HSD [7], NEXUS [8], Dual Parton Model [9], DPMJET [10], String Fusion Model [11, and from other models, such as Linear EXtrapolation of Ultrarelativistic nucleon-nucleon Scattering to Nucleus-nucleus collisions [12], EKRT saturation model [13], Hydrodynamics+UrQMD [14], thermal fireball model [15] and McLerran-Venugopalan model [16]. The assumptions of the underlying QCD dynamics, and even the degrees of freedom (partons, strings, hadrons, classical fields) vary between different models, resulting in a variation of a factor two in the predictions. The multiplicities at $\sqrt{s}=200 \mathrm{AGeV}$ are yet to be measured but, based on the PHOBOS data at 56 and $130 \mathrm{AGeV}$ [17] and an extrapolation to $200 \mathrm{AGeV}$ (the region between the dotted lines), it seems that most models predict too many particles per unit rapidity.

\subsection{Comparison with the first data}

Given the large theoretical uncertainties, the appearance of the first RHIC data on $d N_{\mathrm{ch}} / d \eta$ from Au-Au collisions at $\sqrt{s}=130 \mathrm{AGeV}$ [17] is greeted with a great enthusiasm. The model predictions can now be compared with actual measurements for the first time at collider energies. In Fig. 2, I have shown examples of such comparisons. In LEXUS [18, one first fits all the parameters at nucleon-nucleon level. Extrapolation to ultrarelativistic heavy ion collisions is then done by assuming different dynamics. As nicely illustrated in the figures, the "linear" extrapolation to $A A$, where every binary collision contributes to particle production, gives the best fit at the SPS, whereas the wounded 
nucleon model fits best at both RHIC energies. A suppression mechanism of the produced quanta is also needed in string-based models, such as in the Dual String Model [19], where fusion of strings is needed to fit the RHIC data. HIJING 25] combines pQCD parton dynamics with that of strings. On one hand, a strong gluon shadowing in the hard pQCD component of HIJING reduces the multiplicities, but on the other hand this is partly compensated by jet quenching which feeds some of the jet energy into the system, resulting in the multiplicities close to the measured values [20]. The EKRT saturation model, which is based on pQCD parton dynamics supplemented by a requirement of saturation of produced gluons also predicted correctly both the normalization and the $\sqrt{s}$ scaling for central collisions, as shown by the dotted-dashed line in Fig. 2 (lower left). Another model where the observed multiplicities can be well explained is AMPT, $A$ MultiPhase Transport Model [21], which takes the initial conditions for the partonic and hadronic afterburners from HIJING and fits some of the model parameters from the SPS data. Of the above models only [13] is a published prediction for 56 and $130 \mathrm{AGeV}$ but to my knowledge also [12], [20] (see Fig. 3) and [21] are predictions in the sense that the model parameters were not tuned to fit the first RHIC data.

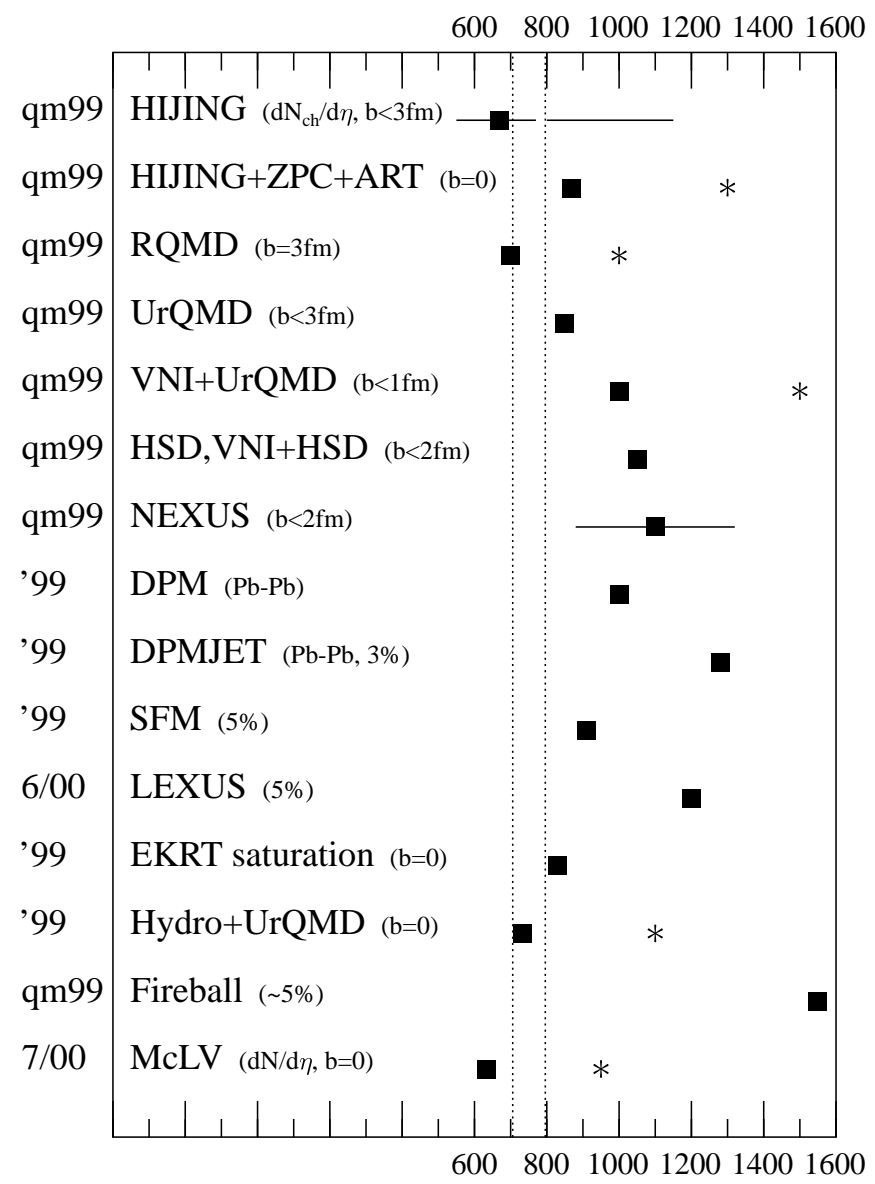

Figure 1. Predictions for $d N_{\mathrm{ch}} / d y$ in $\mathrm{Au}+\mathrm{Au}$ at $\mathrm{y}=0, \sqrt{s}=200 \mathrm{AGeV}$ from different models before the appearance of the first RHIC data. The information in the parentheses specifies the parameters used. The time of releasing the result is indicated on the left. An approximate factor $2 / 3$ has been applied to convert $N_{\text {tot }}$ (asterisks) to $N_{\text {ch }}$ (boxes). Factors 1.1 and 0.9 approximately accounting for the conversion of $\eta$ into $y$ and for a $\sim 5 \%$ centrality selection, respectively, have not been applied in the figure. The vertical dotted lines are obtained by scaling the PHOBOS data at $130 A \mathrm{GeV}$ for $d N_{\mathrm{ch}} / d \eta(6 \%$ central) up by $1.1 *$ $(200 / 130)^{0.37}$ based on the observed $\sqrt{s}$-scaling and to account for the conversion of $\eta$ into $y$. In preparation of this figure I have partly used the review [1].

An implication from the comparison in Fig. 2 is that coherence phenomena in particle production become important at RHIC energies: particle multiplicities are less than what could be expected based on a mere linear extrapolation from SPS to RHIC based on $p p$ physics. It also seems that efficient final state interactions are needed, which in turn 
points towards an early formation of pressure and thermalization. It is also obvious that even within each model the theoretical uncertainties related to the fact that the underlying dynamics is not precisely known in advance, are clearly larger than the experimental (systematic) errorbars of the data. Therefore, the measured multiplicities are indispensable constraints for the models. Essential further constraints, hopefully stringent enough to rule out some models, will be obtained from the other global observable, $d E_{T} / d \eta$, which is more sensitive to the actual evolution of the system than $d N_{\mathrm{ch}} / d \eta$.
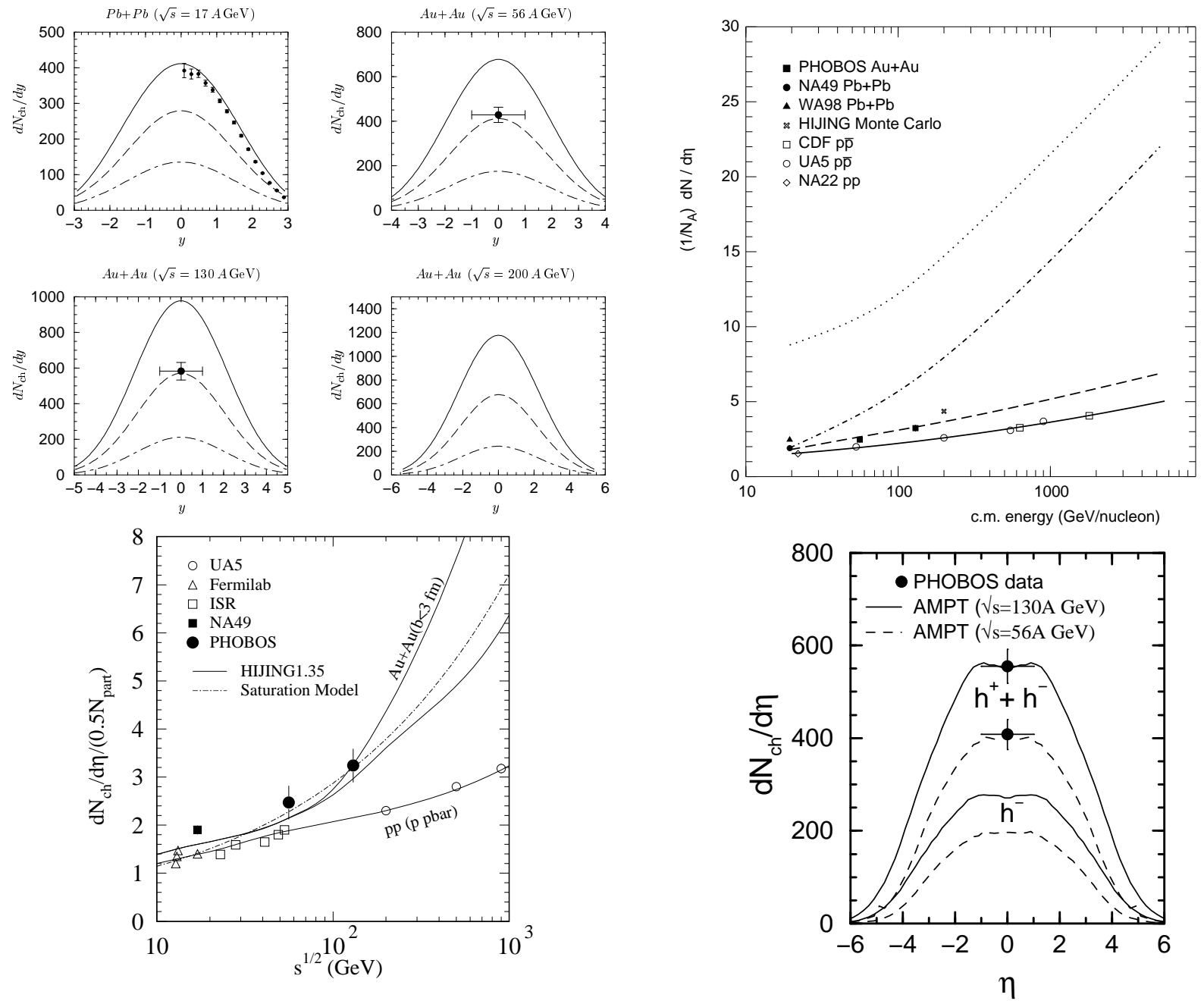

Figure 2. Upper left: LEXUS [18] (solid), wounded nucleon model (dashed); upper right: Dual String Model with (dashed) and without (dotted-dashed) string fusion [19]; lower left: HIJING with shadowing and with and without jet quenching $(\mathrm{Au}+\mathrm{Au}$, solid) [20] together with the EKRT saturation model (dotted-dashed) [13]; Lower right: AMPT model [21].

\subsection{Perturbative QCD in $d N_{\mathrm{ch}} / d \eta$}

Towards higher cms-energies, perturbative production of gluons and quarks in $A A$ collisions becomes more important, eventually even dominant, as first suggested in [23] 26]. The agreement of HIJING and EKRT-type models with the first RHIC data suggest that at RHIC we are witnessing an onset of pQCD particle production.

In first approximation (leading twist), perturbative production of partons, minijets, can be computed assuming factorization of the long- and short-distance physics, i.e. the 
nuclear parton distributions $f_{i}^{A}(x, Q)$ and the partonic cross sections $\hat{\sigma}_{i j}$. Schematically, the integrated minijet cross section for producing partons with transverse momenta larger than a cut-off scale $p_{0}=1 . .2 \mathrm{GeV}$, is $\sigma_{\text {jet }}^{A A}\left(p_{T} \geq p_{0}\right)=K \sum_{i j} f_{i}^{A} \otimes f_{j}^{A} \otimes \hat{\sigma}_{i j}$. So far this has been computed in lowest order pQCD only and a $K$-factor is needed to simulate the NLO contributions. The good news is, however, that due to the recent progress [27, the $K$-factor can now be computed exactly in certain cases, such as in $E_{T}$ production. The order of magnitude is $K \sim 2$, but the exact value depends on $\sqrt{s}$ and on the parton distributions chosen.
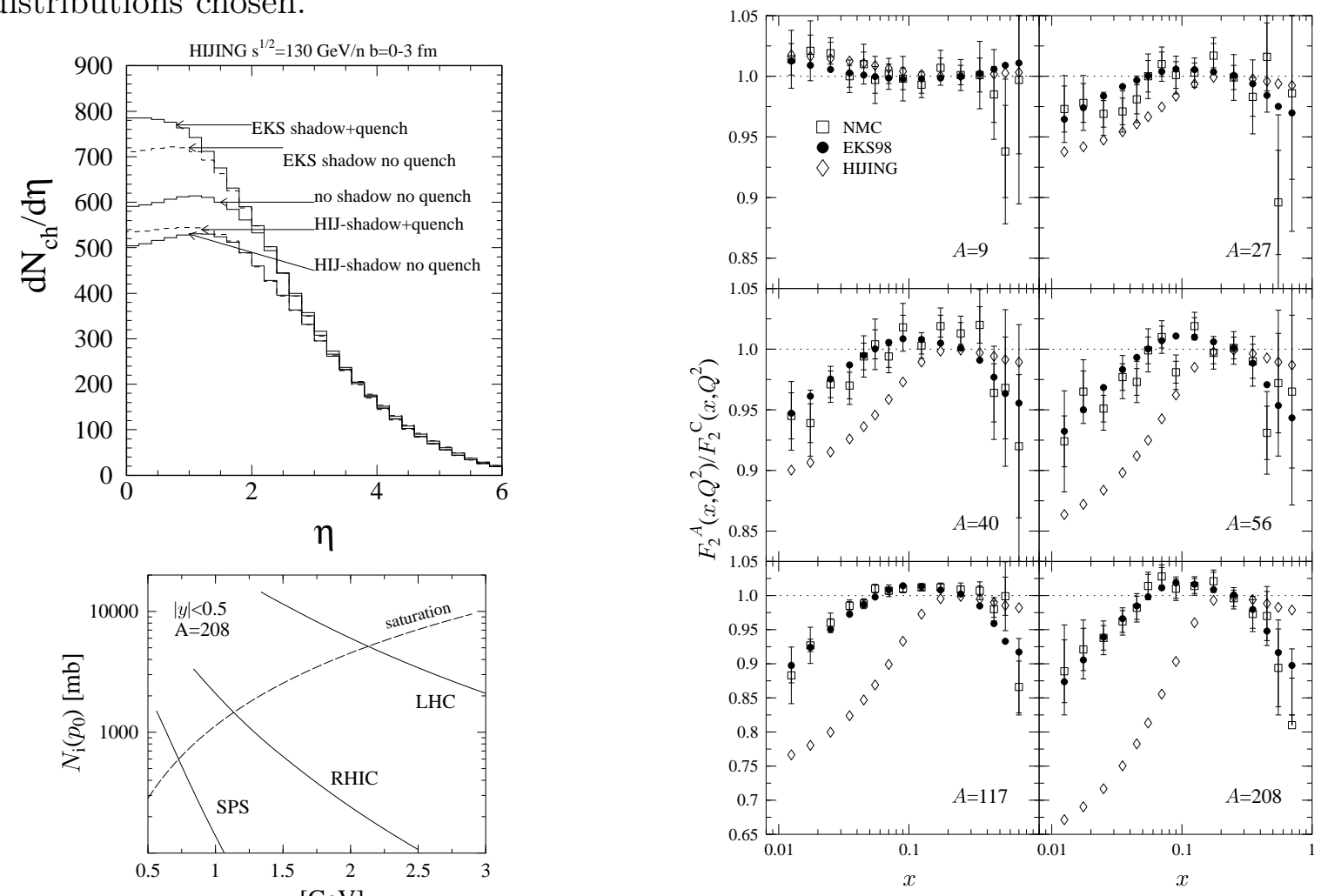

Figure 3. Top left: The HIJING prediction for $d N_{\mathrm{ch}} / d \eta$ in Au-Au collisions at $\sqrt{s}=130$ $A \mathrm{GeV}$ [29]. Bottom left: Determination of the dynamical saturation scale $p_{\text {sat }}=p_{0}(A, \sqrt{s})$ in the EKRT saturation model [13]. $N_{i}$ is the number of produced pQCD partons in $|y| \leq 0.5$. Right: Comparison of the EKS98 nuclear effects (filled circles) [28] and the HIJING default parameterization (open diamonds) with the ratio of structure functions $F_{2}^{A} / F_{2}^{\mathrm{Sn}}$ measured by NMC [30] (open boxes with errorbars).

The largest uncertainty in minijet production is the strong dependence on the minimum transverse momentum scale $p_{0}$ allowed. In two-component models $p_{0}$ determines the division into the pQCD and non-perturbative particle production. In e.g. HIJING [25], $p_{0}=2 \mathrm{GeV}$ (with $K=2$ ) is determined from the $p p$ multiplicity data, and it is kept fixed in the extrapolation to $A A$. This makes the perturbative component to scale as $N \sim A^{4 / 3}$. In the pQCD models supplemented by the requirement of gluon saturation [23,113, $p_{0}$ is determined dynamically as the scale which dominates all particle production in $A A$ collisions. This leads to $p_{\text {sat }}=p_{0}(\sqrt{s}, A)$, as shown in Fig. 3 (bottom left, the intersections of the curves) for the EKRT saturation model.

For the modifications of the parton distributions in nuclei, $f_{i}^{A}(x, Q)=R_{i}^{A}(x, Q) f_{i}(x, Q)$, 
different parametrizations are available. The minimum requirement is that a relevant parametrization is consistent with the existing extensive data in deeply inelastic $l A$ scattering. If collinear factorization is used, as usually is the case in models with minijets, the nuclear modifications should also be compatible with pQCD scale evolution. The EKS98 nuclear parton distributions [28], now included also in the CERN-PDFLIB, fulfill these requirements. An example of the constraints imposed by the DIS data is shown in Fig. 3 . The effects caused by different nuclear parton distributions in the computation of the multiplicities in HIJING is shown by Fig. 3 (top left).

\subsection{Gluon saturation in the initial and final state}

In modeling initial particle production in $A A$ collisions at collider energies, more emphasis has been recently given to models with gluon saturation. The idea can be sketched briefly as follows: let $N_{g}(Q, \Delta Y)$ gluons appear at a scale $Q$ within a rapidity correlation length $\Delta Y$. Saturation of the number of gluons in the transverse plane takes place when $N_{g}(Q, \Delta Y) \times \sigma_{g}(Q) \sim \pi R_{A}^{2}$, where $\sigma_{g}(Q) \sim \alpha_{s}\left(Q^{2}\right) / Q^{2}$ is a cross section for $2 \rightarrow 1$ process and $\pi R_{A}^{2}$ is the available total transverse area. Then, provided that $N_{g}(Q, \Delta Y)$ and $\sigma_{g}(Q)$ can be computed, the saturation scale $Q_{\text {sat }}=Q$ which fulfills the saturation criterion, can be found. In the case of heavy nuclei at high cms-energies, $N_{g}$ becomes large and saturation scales become $Q_{\text {sat }} \gg \Lambda_{\mathrm{QCD}}$.

Gluon saturation in the initial state, in the wave functions of the colliding objects, was first discussed in [31] for $p p$ and in 22, 23 for $A A$ collisions. The idea of initial state saturation is present also in the classical field approach [32.33], where $Q_{\text {sat }}^{2}=$ $\frac{8 \pi^{2} N_{c}}{N_{c}^{2}-1} \alpha_{s} x G\left(x, Q_{\text {sat }}^{2}\right) T_{A}(s)$ with the gluon distribution $x G$ and the nuclear overlap function $T_{A}(s)$. The saturation in the final state is obtained through the initial state gluon saturation, as the transverse profile of the rapidity density of produced gluons in a central $A A$ collision can be expressed as [33] $d N_{g}^{A A} / d^{2} s d y=c C_{F} Q_{\text {sat }}^{2} /\left(\alpha_{s} 2 \pi^{2}\right)$. The "gluon liberation constant" $c$ is expected to be of the order of one [33]. Analytical calculations in the semiclassical approximation [34] give $c=1.39$, and it is interesting to note that if an agreement with the PHOBOS data is required, one gets $Q_{\mathrm{sat}}^{2}=2.1 \mathrm{GeV}^{2} \gg \Lambda_{\mathrm{QCD}}^{2}$. The $\mathrm{SU}(2)$ lattice formulation of the classical field approach [35] gives $c=1.29 \pm 0.09$, the SU(3) calculations are in progress. Similar values are also obtained in [36], based on fits to the PHOBOS data.

Saturation in the final state, i.e. of produced gluons is also possible, even without the requirement of saturation in the initial state. In the EKRT saturation model [13] the

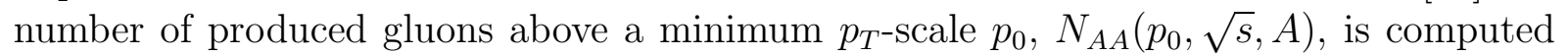
from pQCD. The cross section in the saturation criterion is simply taken as the geometrical one, $\sigma_{g}=\pi / p_{0}^{2}$, not specifying any powers of $\alpha_{s}$ or group theoretical factors. The saturation criterion in a central rapidity unit of central collisions, $N_{A A}\left(p_{0}, \sqrt{s}\right) \times \pi / p_{0}^{2}=$ $\pi R_{A}^{2}$, then determines the dominant scale $p_{\text {sat }}=p_{0}(\sqrt{s}, A)$ as shown in Fig. 3. At a scaling limit $\sigma_{\text {jet }} \sim p_{0}^{-2}$, the saturation scale behaves as $p_{\text {sat }}^{2} \sim A^{1 / 3}$, causing the initial state multiplicity to grow as $N_{A A} \sim A$ instead of $A^{4 / 3}$. A full calculation with realistic parton distributions accounting for the small- $x$ increase and the EKS98 nuclear effects [28] gives a multiplicity of produced partons as [13] $N_{A A}\left(p_{\text {sat }}\right)=1.383 A^{0.922}(\sqrt{s})^{0.383}$.

\footnotetext{
${ }^{1}$ The suppression of the production of small- $p_{T}$ quanta, leading to the saturation, can also be viewed in terms of a screening mass generated into the produced system [39].
} 
Also the released transverse energy, $E_{T i}^{A A}\left(p_{\text {sat }}\right)$ at saturation can be computed from pQCD. As the formation time of the produced system is $\tau_{i} \sim 1 / p_{\text {sat }}$, the initial densities in the EKRT approach can be computed through converting $E_{T i}^{A A}\left(p_{\text {sat }}\right)$ into a thermal energy density $\epsilon_{i}$, which further converts into a thermal number density $n_{i}^{\text {th }}$. On the other hand, conversion of the initial number of gluons at saturation, $N_{A A}\left(p_{\text {sat }}\right)$, into $n_{i}$ gives densities very close to the thermal $n_{i}^{\text {th }}$. In this sense the system looks thermal already at saturation [13]. Thus assuming thermalization at $\tau_{i}$, and an isentropic expansion stage described in terms of (boost invariant) hydrodynamics, the final state multiplicity of charged particles is computable from the entropy produced initially at $\tau_{i}$. This leads to a simple scaling law [13] $d N_{\mathrm{ch}} / d y \approx \frac{2}{3} \times \frac{3.6}{4} N_{i}=0.83 A^{0.922}(\sqrt{s})^{0.383}$ for central collisions. Agreement with the PHOBOS data is amazingly good, as seen in Fig. 2 (lower left). More detailed description of the expansion stage in terms of hydrodynamics with transverse flow effects and resonance decays has now also been done [37], the results are shown in Fig. 4.

Figure 4. $d N_{\mathrm{ch}} / d \eta$ and $d E_{T} / d \eta$ averaged in $|\eta| \leq 1$ vs. $\sqrt{s}$, as obtained from $\mathrm{pQCD}+$ saturation $+2 \mathrm{~d}$ hydrodynamics+resonance decays [37. The open symbols are for central collisions, the closed ones are with a $6 \%$ centrality selection. In the left panel, the lines are from [13] for central collisions, the open circles are the PHOBOS data [17].
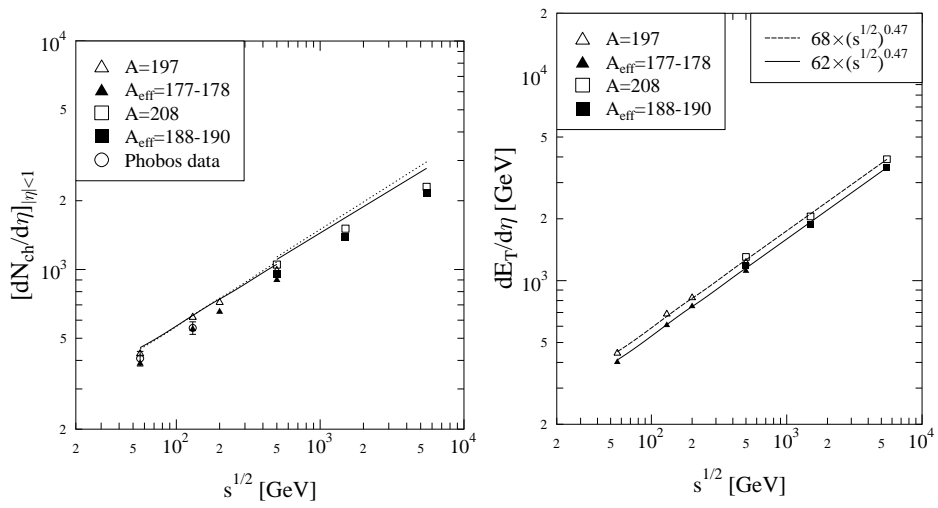

\section{Centrality dependence of $d N_{\mathrm{ch}} / d \eta$}

The dependence of $d N_{\mathrm{ch}} / d \eta$ on the centrality of the collision has been suggested in [20] for testing the predictions from different models. Before the release of the PHENIX data on the centrality dependence, three concrete predictions were submitted:

1. In HIJING [20] the multiplicity is formed as a combination of a component which scales as the number of participants $\sim A$, and a pQCD component which scales with the number of binary collisions $\sim A^{4 / 3}$ (keeping $p_{0}$ fixed): $d N_{\mathrm{ch}}(\mathbf{b}) / d \eta=N_{\text {part }}(\mathbf{b}) n_{\text {soft }}+$ $f N_{\text {bin }}(\mathbf{b}) \sigma_{\text {jet }}^{A A}\left(p_{0}\right)$

2. The EKRT saturation model [13] can be extended to describe the saturation of gluons locally at a transverse distance $\mathbf{s}$ in a collision with an impact parameter $\mathbf{b}$. Assuming the extreme case that saturation fully dominates the particle production, one obtains [38] $d N_{\mathrm{ch}}(\mathbf{b}) / d \eta \approx 0.9 \times 0.9 \times \frac{2}{3} \times \int d^{2} \mathbf{s} p_{\mathrm{sat}}^{2}(\sqrt{s}, A, \mathbf{b}, \mathbf{s}) / \pi$.

3. Dividing the particle production into a "hard" and a "soft" components which scale as $\sim N_{\text {part }}$ and $\sim N_{\text {bin }}$, correspondingly, one may write [36] $d N_{\text {ch }}(\mathbf{b}) / d \eta=x n_{p p} N_{\text {part }}(\mathbf{b})+$ $(1-x) n_{p p} N_{\text {bin }}(\mathbf{b}) / 2$. The average particle multiplicity in $n_{p p}$ is obtained from the $p p$ data, and the fraction $x \sim 0.05 \ldots 0.09$ is obtained by fits to the PHOBOS data [17]. In [36], also an extension of the initial state saturation model [33] to noncentral collisions was suggested in the form $d N_{\mathrm{ch}} / d \eta=\frac{2}{3} c N_{\text {part }} x G\left(x, Q_{\text {sat }}(\mathbf{b})^{2}\right)$, with $c$ obtained from a fit to PHOBOS data.

As shown in Fig. 5 (lower right), the centrality data measured by PHENIX 40 lie in between the EKRT type saturation model and HIJING, quite close to the behaviour 

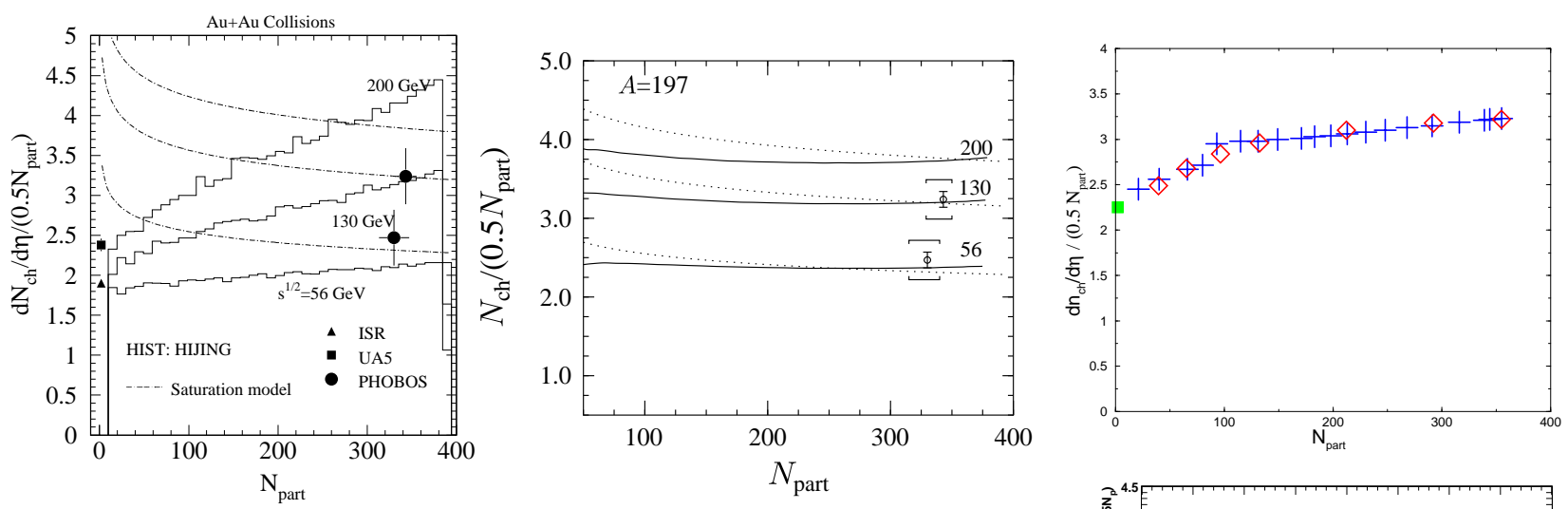

Figure 5. Multiplicity $d N_{\mathrm{ch}} / d \eta /\left(0.5 N_{\text {part }}\right)$ vs. $N_{\text {part }}$ at $\sqrt{s}=130 A \mathrm{GeV}$. From left: HIJING [20] (histograms); local saturation model [38] (solid) and EKRT saturation 13] (dotted); "hard" +"soft" eikonal approach (crosses) and initial state saturation model (diamonds) from [36]. Lower right: the PHENIX data 40.

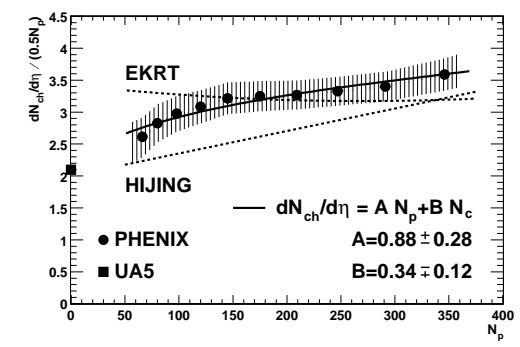

obtained in [36]. Obviously, gluon saturation (EKRT) is not the dominant mechanism for non-central $\left(N_{\text {part }} \lesssim 200\right)$ collisions at $\sqrt{s}=130 \mathrm{AGeV}$ but a non-saturated component is needed at small transverse densities. For central collisions, however, gluon saturation is not ruled out as the dominant mechanism, as also indicated by the PHOBOS data.

The multiplicity measurements alone are not sufficient to rule out models, as most models can be tuned to fit the measured multiplicities and even their centrality dependence. For instance, HIJING has theoretical uncertainties in the division into soft and hard physics and in the extrapolation to $A A$, and also in the pQCD component as discussed above. The saturation models in turn suffer from uncertainties related to the saturation criterion which may be of non-perturbative nature and contain unknown constants and powers of (running) $\alpha_{s}$ which may need to be phenomenologically determined. In any case, it is unlikely that the true theoretical errors in any of the models could be squeezed below the $\pm 10 \%$ level already reached by the first RHIC data.

Of the global variables, $E_{T}$ should be more efficient in ruling out models than $N_{\mathrm{ch}}$, as $E_{T}$ is much more sensitive to the evolution of the system: as shown in [13,37, the ratio of the initially released $E_{T}$ (at saturation) to the measurable final state $E_{T}$ is roughly a factor three. The reduction is due to the $p d V$ work during the expansion stage. Such reduction does not take place in models which do not describe the final state interactions (e.g. HIJING, apart form jet quenching). Also interestingly, the amount of $E_{T}$ released in the classical field model [35] is twice the initial $E_{T}$ obtained in [13], so obviously a very strong energy loss would be needed in order to fall near the measurements of $E_{T}$ shown by PHENIX in this conference.

\section{Elliptic flow}

In non-central collisions, the anisotropic flow has been suggested as a signature of formation of pressure and as a probe of the early stages of the produced system 41]. Pressure can only be formed if the produced particles interact with each other. In noncentral collisions the spatial azimuthal asymmetry of the production zone is transmitted 
to the pressure gradients which drive the transverse flow. In non-central collisions, the initially generated flow is asymmetric, $\left\langle v_{x}^{2}\right\rangle \neq\left\langle v_{y}^{2}\right\rangle$, leading eventually to an asymmetry in the azimuthal particle distributions. The harmonic coefficients of the Fourier expansion of the particle spectra are a quantitative measure of this asymmetry. In particular, the elliptic flow is characterized by the coefficient $v_{2}$, defined as

$v_{2}(y)=\frac{\int d \phi \cos (2 \phi) \frac{d N}{d y d \phi}}{\int d \phi \frac{d N}{d y d \phi}} \quad$ and $\quad v_{2}\left(y, p_{T}\right)=\frac{\int d \phi \cos (2 \phi) \frac{d N}{d y d p_{T}^{2} d \phi}}{\int d \phi \frac{d N}{d y d p_{T}^{2} d \phi}}$

Clear elliptic flow signal, up to $6 \%$ for $v_{2}$ and up to $15 \%$ for $v_{2}\left(p_{T}\right)$ in minimum bias events in $\mathrm{Au}-\mathrm{Au}$ at $\sqrt{s}=130 \mathrm{AGeV}$, has now been measured at RHIC by STAR 42 (shown in Fig. 7). An attempt to briefly summarize the situation with the semiclassical cascade models is presented in Fig. 6, where $v_{2}$ is shown from RQMDv2.4, UrQMD and $\mathrm{ZPC}$ at $\sqrt{s}=200 \mathrm{AGeV}$ (the measured $v_{2}$ can be expected to be close to that at $\sqrt{s}=200$ $A \mathrm{GeV}$ 47]): Partonic cascades can in principle generate large enough $v_{2}$ but very large rescattering cross sections are needed [43]. As the mean-free paths of particles then become very short, this speaks in favour of hydrodynamics. Hadronic cascades predict the linear rise of $v_{2}\left(p_{T}\right)$ at $p_{T} \lesssim 500 \mathrm{MeV}$ but they level off too soon after that [44]. These models do not seem to produce sufficient $p_{T}$-averaged $v_{2}$ either [44, 45], which speaks in favour of an early formation of collectivity and pressure in the partonic system.
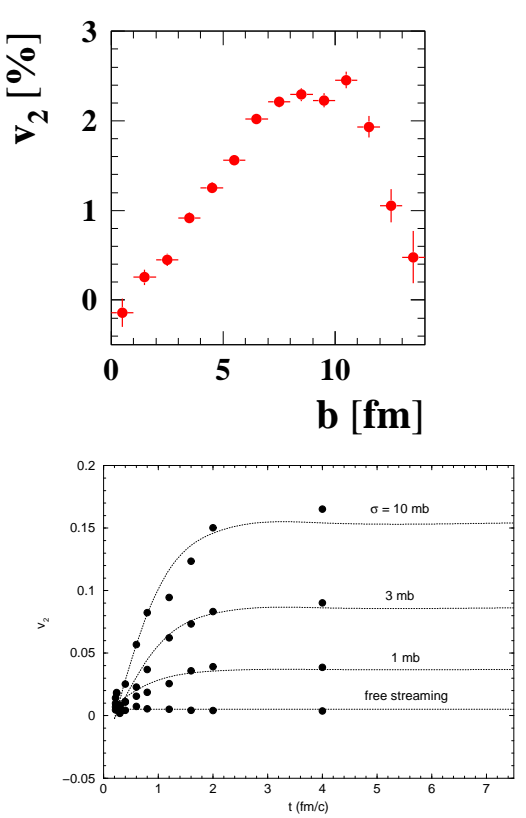
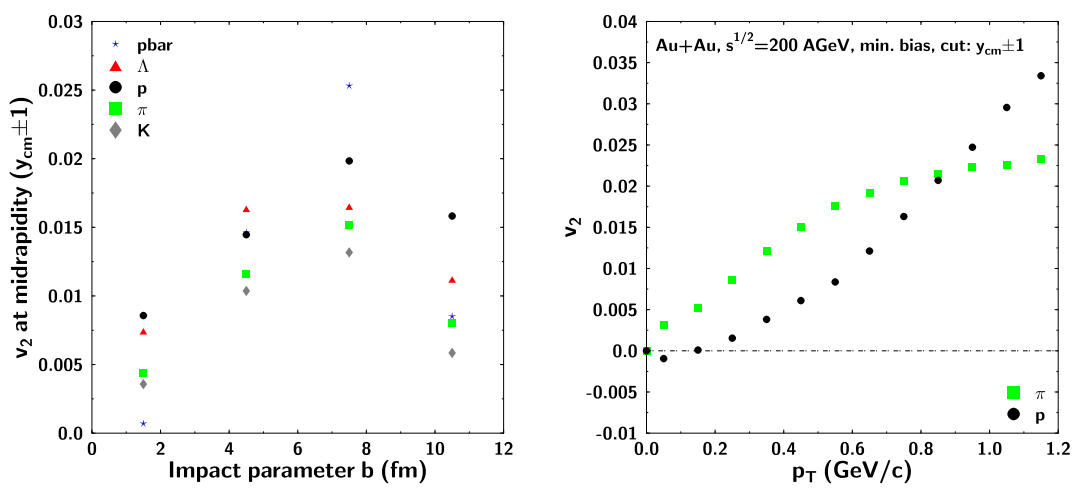

Figure 6. Elliptic flow for $\mathrm{Au}-\mathrm{Au}$ at $\sqrt{s}=200 \mathrm{AGeV}$ from cascade models. From upper left: $v_{2}$ vs. $\mathbf{b}$ from RQMDv2.4 45], $v_{2}$ vs. b from UrQMD 44, and $v_{2}\left(p_{T}\right)$ vs. $p_{T}$ from UrQMD vs. $p_{T}$ [4]. Lower panel: $v_{2}$ vs. time from the ZPC parton cascade 43.

The observed strong elliptic flow confirms the prediction based on hydrodynamics 477. Detailed comparisons have also been done, as shown in Fig. 7. The $v_{2}$ obtained in the "conventional" hydrodynamic approach 48, where the initial conditions are constrained by the observed final state multiplicity and where the Cooper-Frye procedure 46] is applied for the decoupling, agrees with the data quite well. Similar conclusion is obtained in the Hydro-to-Hadrons approach [49], where the RQMDv2.4 hadron cascade is switched on below $T=160 \mathrm{MeV}$ in order to treat the chemical and kinetic freeze-out in more detail. At very large impact parameters, where application of a purely hydrodynamical 
system may be questioned in any case, these approaches seem to produce too much $v_{2}$. Also $v_{2}\left(p_{T}\right)$ in the conventional hydrodynamic approach agrees with the STAR data very well up to $2 \mathrm{GeV}$ or so. The deviation at high $p_{T}$ is not surprising, since in a finite dynamic system with a finite lifetime the large- $p_{T}$ tails will not be adequately described by hydrodynamics. Another interesting observation is that the amount of $v_{2}$ is not very sensitive to the equation of state (EoS) used. Therefore, the single particle spectra are needed to constrain the remaining uncertainties such as the EoS.
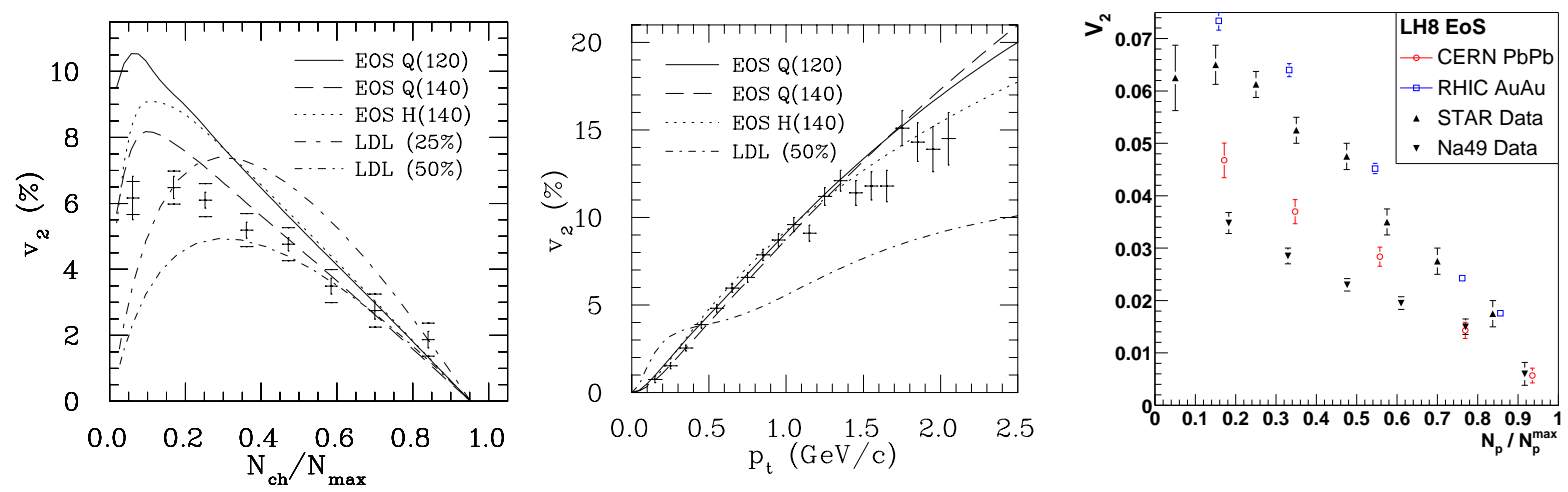

Figure 7. From left: $v_{2}$ and $v_{2}\left(p_{T}\right)$ from hydrodynamics [48] and data from STAR [42]. On right: $v_{2}$ from hydrodynamics followed by RQMDv2.4 [49].

In conclusion, the first results from RHIC have again demonstrated the importance of a close interplay between theory and experiments: predictions for observables are difficult to obtain from truly first principles. Indispensable constraints for the models are obtained from certain sets of observables, such as the ones considered here. Especially useful will be the studies of the systematics in $\sqrt{s}, A$ and $\mathbf{b}$. When the production and evolution stage of the strongly interacting system are theoretically under control, the signals of the QGP can be predicted and found.

\section{Acknowledgements}

I thank K. Tuominen, K. Kajantie, P. Huovinen, N. Armesto, and V. Ruuskanen for help in preparing this talk, V.J. Kolhinen for help with the eps-figures, X.-N. Wang for sending me Fig. 3a, H. Honkanen for preparing Fig. 3c, and the Academy of Finland for financial support.

\section{REFERENCES}

1. S.A. Bass et al., Proc. Quark Matter '99, Nucl. Phys. A 661 (1999) 205c.

2. X.-N. Wang, in [1].

3. B. Zhang, in [1].

4. H. Sorge, nucl-th/9905008, also in Quark Matter '99.

5. M. Bleicher, in [1].

6. S.A. Bass, in 11.

7. W. Cassing, in [1].

8. H.J. Drescher, in [1].

9. A. Capella, A. Kaidalov and J. Tran Thanh Van, Heavy Ion Phys. 9 (1999) 169 [hepph/9903244.

10. J. Ranft, hep-ph/9911213. 
11. N. Armesto and C. Pajares, Int. J. Mod. Phys. A 15 (2000) 2019 hep-ph/0002163].

12. S. Jeon and J. Kapusta, "LEXUS", contest on the RHIC predictions, June 2000.

13. K.J. Eskola, K. Kajantie, P.V. Ruuskanen and K. Tuominen, Nucl. Phys. B 570 (2000) 379 hep-ph/9909456.

14. S.A. Bass et al, Phys. Rev. C 60 (1999) 021902 [nucl-th/9902062]; A. Dumitru in [1].

15. J. Stachel, in [1].

16. A. Krasnitz and R. Venugopalan, Phys. Rev. Lett. 86 (2001) 1717 [hep-ph/0007108].

17. B.B. Back et al. [PHOBOS Coll.], Phys. Rev. Lett. 85 (2000) 3100 hep-ex/0007036].

18. S. Jeon and J. Kapusta, Phys. Rev. C 63 (2001) 011901 nucl-th/0009032.

19. J. Dias de Deus and R. Ugoccioni, Phys. Lett. B 491 (2000) 253 hep-ph/0008086.

20. X.-N. Wang and M. Gyulassy, nucl-th/0008014.

21. Z. Lin, S. Pal, C.M. Ko, B. Li and B. Zhang, nucl-th/0011059; Z. Lin, these proceedings.

22. A. H. Mueller and J. Qiu, Nucl. Phys. B 268 (1986) 427.

23. J.P. Blaizot and A.H. Mueller, Nucl. Phys. B 289 (1987) 847.

24. K. Kajantie, P.V. Landshoff and J. Lindfors, Phys. Rev. Lett. 59 (1987) 2527;

K.J. Eskola, K. Kajantie and J. Lindfors, Nucl. Phys. B 323 (1989) 37.

25. X.-N. Wang and M. Gyulassy, Phys. Rev. D 44 (1991) 3501.

26. K. Geiger and B. Müller, Nucl. Phys. B 369 (1992) 600.

27. K.J. Eskola and K. Tuominen, hep-ph/0010319, Phys. Rev. D, in press;

A. Leonidov and D. Ostrovsky, hep-ph/9811417.

28. K.J. Eskola, V.J. Kolhinen and P.V. Ruuskanen, Nucl. Phys. B 535 (1998) 351 hepph/9802350]; K.J. Eskola, V.J. Kolhinen and C.A. Salgado, Eur. Phys. J. C 9 (1999) 61 hep-ph/9807297.

29. X.-N. Wang, private communication.

30. M. Arneodo et al. [New Muon Coll.], Nucl. Phys. B 481 (1996) 23.

31. L.V. Gribov, E.M. Levin and M.G. Ryskin, Phys. Rept. 100 (1983) 1.

32. L. McLerran and R. Venugopalan, Phys. Rev. D 49 (1994) 2233 [hep-ph/9309289]; ibid. 3352 hep-ph/9311205]; Phys. Rev. D 50 (1994) 2225 [hep-ph/9402335]; R. Venugopalan, these proceedings.

33. A.H. Mueller, Nucl. Phys. B 572 (2000) 227 hep-ph/9906322.

34. Y.V. Kovchegov, hep-ph/0011252; Y.V. Kovchegov, these proceedings.

35. A. Krasnitz and R. Venugopalan, Phys. Rev. Lett. 86 (2001) 1717 hep-ph/0007108.

36. D. Kharzeev and M. Nardi, nucl-th/0012025.

37. K.J. Eskola, P.V. Ruuskanen, S.S. Räsänen and K. Tuominen, JYFL-3/01, hep-ph/0104010.

38. K. J. Eskola, K. Kajantie and K. Tuominen, Phys. Lett. B 497 (2001) 39 hep-ph/0009246.

39. K. J. Eskola, B. Müller and X. Wang, Phys. Lett. B 374 (1996) 20 hep-ph/9509285].

40. K. Adcox et al. [PHENIX Coll.], nucl-ex/0012008.

41. J.Y. Ollitrault, Phys. Rev. D46 (1992) 229; Nucl. Phys. A638 (1998) 195c.

42. K.H. Ackermann et al. [STAR Coll.], Phys. Rev. Lett. 86 (2001) 402.

43. B. Zhang, M. Gyulassy and C.M. Ko, Phys. Lett. B 455 (1999) 45 nucl-th/9902016; see D. Molnar in [1], and these proceedings.

44. M. Bleicher and H. Stöcker, hep-ph/0006147.

45. R.J. Snellings, A.M. Poskanzer and S.A. Voloshin, nucl-ex/9904003.

46. F. Cooper and G. Frye, Phys. Rev. D 10 (1974) 186.

47. P.F. Kolb, J. Sollfrank and U. Heinz, Phys. Rev. C 62 (2000) 054909 [hep-ph/0006129].

48. P.F. Kolb, P. Huovinen, U. Heinz and H. Heiselberg, Phys. Lett. B 500 (2001) 232 hepph/0012137]; P. Huovinen, these proceedings.

49. D. Teaney, J. Lauret and E.V. Shuryak, nucl-th/0011058; D. Teaney, these proceedings. 